therefore, PGA trans-eliminase, at least in A. liquefaciens, is a constitutive enzyme.

Hsu and Vaughn's results also indicate that PGA trans-eliminase is catabolite repressible, and further experiments demonstrated that various mono, di and polysaccharides could function as potent repressors. The repressor produced in cultures grown on unlimited PGA has not been identified, but, although it could be an unsaturated monogalacturonic acid, it is not D-galacturonic. Hsu and Vaughn favour a subsequent metabolite such as 2-keto-3-deoxy-D-gluconic acid as the actual repressor. The catabolic repressibility of PGA trans-eliminase was substantiated by isolating a mutant that produced the enzyme constitutively in the presence of excess carbon substrates. This study of PGA trans-eliminase is important as the first finding of an extracellular depolymerase that is under the control of catabolite repression. The implications of this investigation are clear and the re-examination of other degradative enzyme systems such as cellulase could provide interesting results for those concerned with both enzyme production and microbial ecology.

\section{ACTIVE SITES}

\section{Simple and Fast}

\section{from our Enzymology Correspondent}

Carbonic anhydrase is a doubly distinguished enzyme. It catalyses what is perhaps the simplest biological reaction, the addition of carbon dioxide to water to give bicarbonate ion, and it is the fastest known enzyme of its type. Each of its active sites goes through the reaction cycle $10^{5}$ times per second. The mechanism of the enzyme is therefore of great interest, and S. Bradbury of Harvard describes an attractive study of the topic in the latest issue of the Journal of Biological Chemistry (244, 2002; 1969).

A histidine residue and a zinc atom are known to be at or near the enzyme's active site, and Bradbury explored the relationship of these groups in terms of their reactivity towards iodoacetate, a useful target reagent for many enzyme nucleophiles. Iodoacetate reversibly inhibited carbonic anhydrase $B$ from human red cells, and the inhibition turned out to be strongly $p \mathrm{H}$-dependent. The inhibition lessened as $p \mathrm{H}$ rose, with a point of inflexion near $p \mathrm{H} 7 \cdot 0$. This result suggests that iodoacetate, which is itself negatively charged, inhibits the enzyme by binding to an active site group that also acquires a negative charge around $p \mathrm{H} 7 \cdot 0$.

Bradbury assumes that the group in question is a hydrated version of the active site zinc atom, losing a proton at $p H 7 \cdot 0$, and there is indeed good evidence that various anions bind to the zinc of the enzyme. Iodoacetate also irreversibly inhibited the enzyme, and amino-acid analysis of the irreversibly inhibited enzyme showed that iodoacetate had become covalently linked to an enzyme histidine residue. The rate of loss of enzyme activity was the same as the rate of appearance of the histidine-iodoacetate adduct, and sulphanilamide, which binds to the active site of the enzyme, prevented the inhibition. This is to be expected. But it is more surprising that inhibition was never completeit regularly tailed off to an asymptote of 5 per cent of the initial activity-and that in the presence of sufficient urea to denature the enzyme, the reaction of iodoacetate and enzyme histidine was curtailed.
But for this persistent residual activity, it would be easy to frame a plausible mechanism involving the zine atom, to which iodoacetate binds reversibly, and a nearby histidine residue, to which iodoacetate binds covalently. The residual activity makes things more complex, however. Perhaps the histidine which reacts with iodoacetate is irrelevant to the catalytic mechanism, and the iodoacetate merely slows catalysis, by steric or electrostatic hindrance; perhaps the histidine is involved in the workings of the native enzyme, but the enzyme can still limp along without it; perhaps inhibition is not homogeneous throughout the population of enzyme molecules, and the residual 5 per cent of activity represents some side reaction. There are many possibilities, but until more experimental results have been obtained further speculation is perhaps unwarranted.

\section{LIPIDS \\ Abnormalities of Metabolism}

\section{from our Medical Biochemistry Correspondent}

NEw separation techniques have made possible considerable advances in our knowledge of lipid metabolism, and are revealing inheritcd abnormalities in these processes. A familial plasma lecithin: cholesterol acyl transferase (LCAT) deficiency was first described in three Norwegian sisters eighteen months ago. The same condition has been found recently in a sister and brother from Sweden (Brit. Med. J., ii, 283; 1969). All the patients had opacity of the cornea caused by deposits of lipid, they had normochromic anaemia and they excreted protein in the urine although there was no evidence of renal insufficiency. Changes in the kidney are the most serious consequence of this disorder; one of the Swedish cases died from uraemia, and the histological appearance of his kidneys at autopsy was similar to that of a kidney biopsy from one of the Norwcgian sisters. There was almost no plasma LCA'T activity and as a direct consequence very little of the cholesterol in the blood was esterified, there was more plasma lecithin than usual and very little lysolethicin. In all cases the concentration of plasma triglyceride was greater than normal, but it was interesting that there was almost no $\alpha$-lipoprotein. The Swedish patients still had about 27 per cent of their cholesterol in the ester form while the Norwegians had only 3-10 per cent esterified cholesterol. Cholesterol esters had more linoleic acid and less saturated fatty acids in the Swedish patients than in the Norwcgian family.

A better known hereditary disorder of lipid metabolism, called Niemann-Pick disease, is characterized by the accumulation of sphingomyelin in the tissues. Measurements of the sphingomyelin-cleaving enzyme activity of kidney and liver biopsies from these paticnts showed that the condition was probably caused by a deficiency of this enzyme rather than by an abnormality in the synthesis of sphingomyelin. Sloan et al. (Biochem. Biophys. Res. Commun., 34, 582; 1969) have shown that fibroblasts from patients with the two infantile forms of Niemann-Pick disease are also deficient in sphingomyelin-cleaving enzyme activity.

They measured the enzyme activity according to the release of ${ }^{14} \mathrm{C}$-phosphoryl choline from ${ }^{14} \mathrm{C}$-methylsphingomyelin (expressed as units per million cells in 